Anuario Latinoamericano Ciencias Políticas

y Relaciones Internacionales

vol. 7, 2019

pp. 365-366

DOI: $10.17951 /$ al.2019.7.365-366

\section{EI II Congreso Internacional de Derechos Humanos, Justicia y Migración, 11-16 de febrero de 2019, Guadalajara, México}

\section{The 2nd International Congress on Human Rights, Justice and Migration, February 11-16, 2019, Guadalajara, Mexico}

Del 8 al 20 de febrero de 2019, por invitación del grupo internacional de investigación científica Red Internacional de Derechos Humanos y Derecho Internacional Humanitario (REDDIH), la Prof. Dra. Katarzyna Krzywicka, politóloga y latinoamericanista de la Facultad de Ciencias Políticas y Periodismo de la Universidad Maria Curie-Skłodowska, realizó una estancia de investigación en la Universidad de Guadalajara. La Prof. Krzywicka, miembro del Consejo Científico de la REDDIH desde 2018, participó en el Pre-Congreso sobre el tema „Derechos Humanos, Justicia y Migración”, que tuvo lugar del 11 al 12 de febrero. Como parte de este evento, se realizaron elecciones a la Junta Ejecutiva de la REDDIH. La Prof. Krzywicka fue nombrada vicedirectora del Comité Editorial de la REDDIH. Además, participó en las reuniones del Comité Ejecutivo de la REDDIH, cuyo objetivo era desarrollar un programa de acción para el año 2019. La Prof. Krzywicka también fue invitada, como uno de los tres revisores, al Jurado de la Comisión de la REDDIH, para evaluar once trabajos escritos por los doctorandos de la Universidad de Guadalajara, presentados para el concurso sobre derechos humanos, organizado como parte del Pre-Congreso de la REDDIH. El grupo internacional de investigación de la REDDIH está formado por científicos de trece países de América Latina, Europa y Asia: Argentina, Brasil, Chile, Guatemala, España, Israel, Colombia, México, Alemania, Polonia, Estados Unidos, Gran Bretaña e Italia.

La visita a la Universidad de Guadalajara sirvió también para promover la actividad editorial de la Facultad de Ciencias Políticas y Periodismo de la UMCS. Por invitación del Centro Universitario de Ciencias Sociales y Humanidades de la Universidad de Guadalajara, la Prof. Katarzyna Krzywicka, fundadora y editora en jefe de la revista Anuario Latinoamericano - Ciencias Políticas y Relaciones Internacionales, publicada desde 2014 por la Facultad de Ciencias Políticas de la UMCS, participó en la presentación abierta del periódico en la biblioteca principal de la universidad, en la Librería Carlos Fuentes. A la presentación del Anuario Latinoamericano también asistió un miembro del Consejo Científico de la revista, el Prof. Dr. Alberto Rocha Valencia, 
politólogo del Centro de Estudios Latinoamericanos de la Universidad de Guadalajara. La presentación del Anuario Latinoamericano fue inaugurada por el presidente de la REDDIH, José Trinidad Padilla López. El evento fue organizado en cooperación con el Departamento de Estudios Políticos y el Departamento de Estudios Internacionales de la Universidad de Guadalajara.

Durante su estancia en la Universidad de Guadalajara, la Prof. Krzywicka también participó en el II Congreso Internacional de Derechos Humanos, Justicia y Migración, del 15 al 16 de febrero de 2019. El Congreso fue organizado por la REDDIH en cooperación con el Supremo Tribunal de Justicia y el Consejo de la Judicatura del estado de Jalisco, entre otros. Como parte de este evento, el día de la inauguración del Congreso, la Prof. Krzywicka impartió una conferencia sobre „El derecho a la libertad de conciencia y religión en Polonia. Desarrollo histórico en su dimensión institucional y jurídica” durante la sesión plenaria titulada „Los Derechos Humanos en una perspectiva histórica”. La visita en la Universidad de Guadalajara también brindó la oportunidad de presentar el perfil científico de la Facultad de Ciencias Políticas y Periodismo de la UMCS y, en particular, los estudios en el campo de ciencias políticas enfocados en la región de América Latina así como la oferta didáctica para estudiantes internacionales impartida en inglés.

Katarzyna Krzywicka Facultad de Ciencias Políticas y Periodismo Universidad Maria Curie -Skłodowska Lublin, Polonia $\triangle$ katarzyna.krzywicka@umcs.edu.pl https://orcid.org/0000-0001-5623-5384 